\title{
Estimation of Propagation Structure by means of Hopfield Neural Network
}

\author{
Takashi Maruyama Yoshihiko Kuwahara \\ Faculty of Engineering, Shizuoka University
}

\section{Introduction}

In order to develop the spatial-temporal equalization technique for $3 \mathrm{G} / 4 \mathrm{G}$ mobile communication system, it is necessary to grasp indoor or outdoor propagation structure. In recent year, it is proposed to apply the artificial neural network (ANN) to DOA estimation [1]-[2]. The purposes are to direct narrow beam to the terminals after detecting the directions. In this paper, we propose an algorithm using an ANN to estimate coupled DOA and TOA of multiple incident waves.

\section{Algorithm}

In Hopfield neural network shown as Fig.1, the conditions are renewed as energy expressed by next Lyapunov function is minimized [3].

$\mathrm{E}=-\frac{1}{2} \sum_{p=1}^{\mathrm{P}} \sum_{q=1}^{\mathrm{P}} w_{p q} v_{p} v_{q^{-}} \sum_{p=1}^{\mathrm{P}} \mathbf{I}_{p} v_{p}$

And, input signal of each neuron $\mathrm{u}_{\mathrm{p}}$ satisfies with next differential equation.

$\mathbf{R}_{p} \mathbf{C}_{p} \frac{d u_{p}}{d t}=\sum_{q \neq p}^{\mathrm{P}} \mathbf{R}_{p} w_{p q} v_{q}-u_{p}+\mathbf{R}_{p} \mathbf{I}_{p}$

Here, $\mathrm{w}_{\mathrm{pq}}$ is inverse of $\mathrm{R}_{\mathrm{pq}} \cdot \mathrm{v}_{\mathrm{q}}$ is a response of neuron and it is decided by next sigmoid function.

$v_{q}=f\left(u_{q}\right)=\frac{1}{2}\left\{1+\tanh \left(\beta u_{q}\right)\right\}$

Here, $B$ is neuron gain.

In order to estimate propagation parameters, we define category p. A wave subject to category $p$ has delay of $i$ symbols and direction of arrival of $\left(\varphi_{j}, \theta_{k}\right) \cdot \varphi_{j}$ and $\theta_{k}$ are defined $\operatorname{as} \varphi_{\mathrm{j}}=(\mathrm{j}-1) 50 / \mathrm{J}($ deg. $)$ and $\theta_{\mathrm{k}}=(\mathrm{k}-1) 50 / \mathrm{K}$ (deg.), respectively. $\mathrm{J}$ and $\mathrm{K}$ are category numbers of azimuth and elevation angle, respectively. When $\mathrm{N}$ data of frequency domain from a rectangular array of $\mathrm{M}_{1} \times \mathrm{M}_{2}$ elements are used, incident signal $\mathrm{s}_{\mathrm{p}}{ }^{\mathrm{m} 1 \mathrm{~m} 2 \mathrm{n}}$ to the array antenna is expressed by

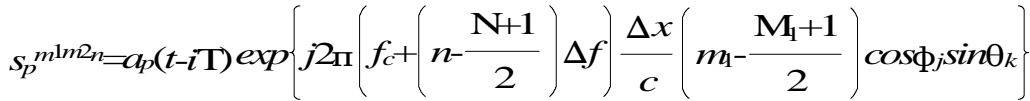

$$
\begin{aligned}
& \exp j 2 \pi\left(f_{c}+\left(n-\frac{\mathrm{N}+1}{2}\right) \Delta f \frac{\Delta y}{c}\left(m_{2^{-}} \frac{\mathrm{M}_{\mathrm{b}}+1}{2}\right) \sin \phi_{j} \sin \theta_{k}\right\}
\end{aligned}
$$

$a_{p}(t)$ is time sequence complex signal, $T$ is symbol rate of time sequence signal, $f_{c}$ is carrier frequency, $\Delta \mathrm{f}$ is frequency spacing, $\Delta \mathrm{x}$ and $\Delta \mathrm{y}$ are element spacing in the 
direction of $\mathrm{x}$ and $\mathrm{y}$-axis. In order to get signal in the frequency domain, we transform time sequence signal to frequency domain by FFT as shown to Fig.2. Quantity of which signal of all the categories contribute to $\left(\mathrm{m}_{1}, \mathrm{~m}_{2}, \mathrm{n}\right)$ th received signal is expressed by next formula.

$x_{m 1 m 2 n}(t)=\sum_{p=1}^{\mathrm{P}} w_{p}^{m 1 m 2 n^{m}} S_{p}^{m 1 m 2 n}(t)$

In case of estimating such parameters as propagation, it is necessary to set each weight $\mathrm{w}_{\mathrm{p}}{ }^{\mathrm{m} 1 \mathrm{~m} 2 \mathrm{n}}$ properly by minimizing the square root error "Es" between received signal vector $\mathrm{y}=\left[\mathrm{y}_{111} \ldots \mathrm{y}_{\mathrm{M} 1 \mathrm{M} 2 \mathrm{~N}}\right]^{\mathrm{T}}$ and vector $\mathrm{x}=\left[\mathrm{x}_{111} \ldots \mathrm{x}_{\mathrm{M} 1 \mathrm{M} 2 \mathrm{~N}}\right]^{\mathrm{T}}[2]$.

$\mathrm{E} s=\llbracket y-x \rrbracket=\operatorname{Re}\left[\sum_{q}^{\mathrm{P}} \sum_{r \neq q}^{\mathrm{P}}\left(s_{q}^{\mathrm{H}_{s}} s_{r} w_{q} w_{r}-\sum_{q}^{\mathrm{P}}\left(2 y^{\mathrm{H}} s_{q}-s_{q}{ }^{\mathrm{H}} s_{r}\right) w_{q}\right]\right.$

Here, Vector $\mathrm{s}_{\mathrm{q}}$ is defined by

$s_{q} \rightleftharpoons\left[s_{q}^{111} \ldots . . s_{q}^{m 1 m 2 n} \ldots . . . s_{q}^{\mathrm{MINEN}}\right]^{\mathrm{T}}$

From Formula (1) and (6), weight $\mathrm{w}_{\mathrm{qr}}$ and $\mathrm{I}_{\mathrm{q}}$ can be expressed by next formulas.

$w_{q r}=-\operatorname{Re}\left[s_{q}{ }^{\mathrm{H}} s_{r}\right] \quad \mathrm{I}_{q}=\operatorname{Re}\left[y^{\mathrm{H}} s_{q}-\frac{1}{2} s_{q}{ }^{\mathrm{H}} s_{r}\right]$

$\mathrm{w}_{\mathrm{qr}}$ makes zero when $\mathrm{q}=\mathrm{r}$. Weight matrix among neurons is expressed by next formula

$\mathrm{W}=\left(\begin{array}{llll}0 & w_{12} & \ldots & w_{1 \mathrm{P}} \\ w_{21} & 0 & \ldots & w_{2 \mathrm{P}} \\ \ldots & \ldots & \ldots & \ldots \\ w_{\mathrm{P} 1} & w_{\mathrm{P} 2} & \ldots & 0\end{array}\right)$

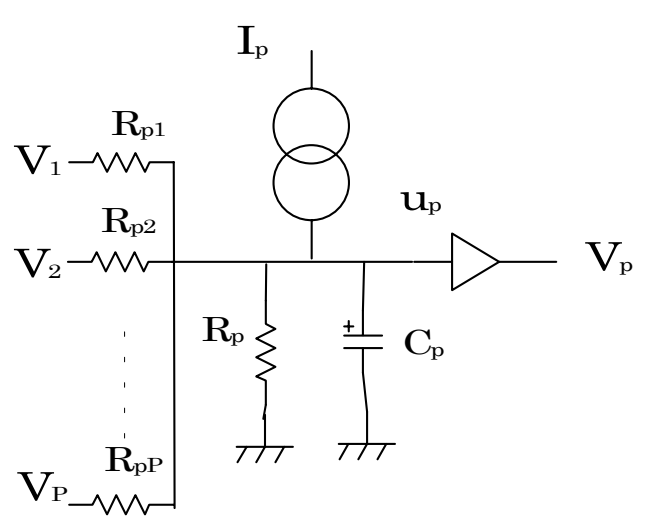

Fig.1 Hopfield neural network

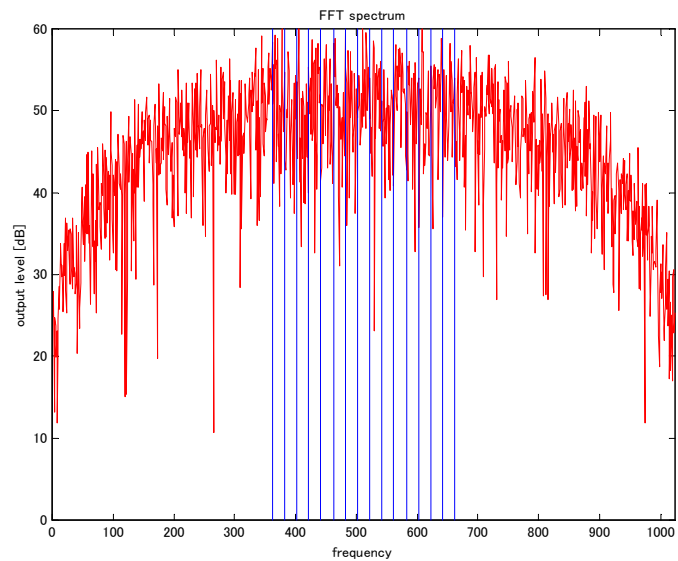

Fig.2 signal in the frequency domain

\section{Simulation}

We have defined 180 categories, that is $(I, J, K)=(6,6,5)$. And we have used signal modulated PN9 sequence by QPSK as the incident wave. 10 sampling points in the frequency domain received by $8 \times 8$ elements planer array antenna have been used for input data. Fig3 shows one example of coupled DOA and TOA estimation results. In 
this case, 2 waves with $(\varphi, \theta, \mathrm{T})=\left(10^{\circ} 40^{\circ} 0\right.$ symbols $)$ and $\left(30^{\circ} 40^{\circ} 1\right.$ symbols $)$ are incident. Then, category parameters $(\mathrm{i}, \mathrm{j}, \mathrm{k})$ are $(1,2,5)$ and $(2,4,5)$, respectively. 2 neurons subject to each category ignite. Fig.4 shows another example of 3 incident waves with with $(\varphi, \theta, \mathrm{T})=\left(0^{\circ} 0^{\circ} 0\right.$ symbols $),\left(0^{\circ} 30^{\circ} 2\right.$ symbols $)$ and $\left(20^{\circ} 0^{\circ} 1\right.$ symbols $)$.

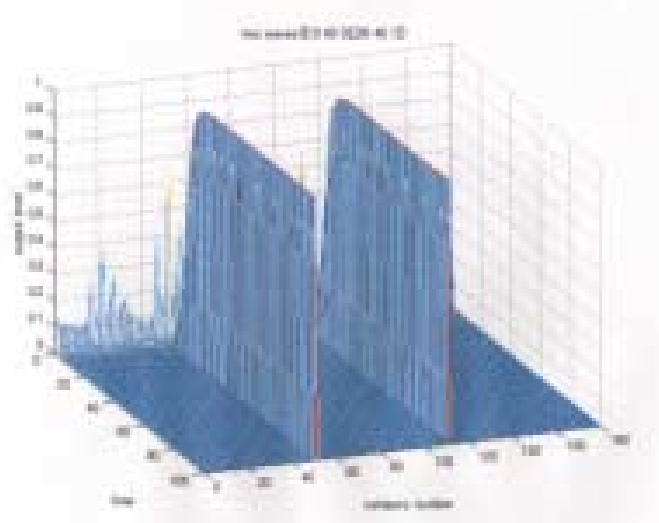

Fig.3 Estimation result $\left(10^{\circ} 40^{\circ} 0\right.$ symbols $)$ $\left(30^{\circ} 40^{\circ} 1\right.$ symbols $)$

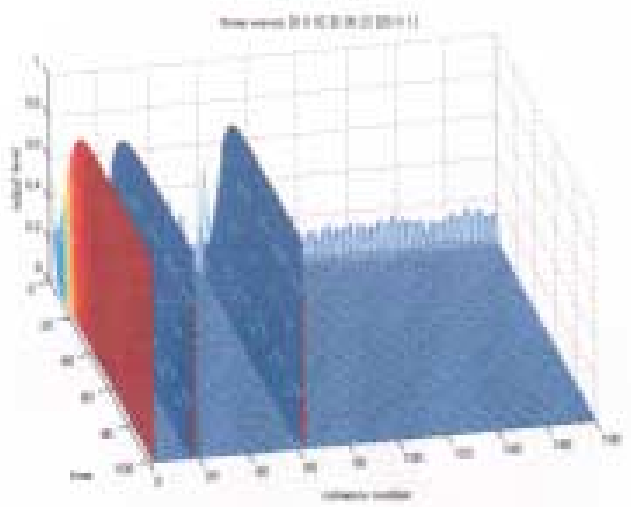

Fig.4 Estimation result $\left(0^{\circ} 0^{\circ} 0\right.$ symbols $),\left(0^{\circ} 30^{\circ} 2\right.$ symbols $)$ $\left(20^{\circ} 0^{\circ} 1\right.$ symbols $)$.

Category number $\mathrm{P}$ can be expressed by next formula.

$\mathrm{P}=(j-1) * 30+(k-1) * 5+i$

We have evaluated how to ignite neurons in the case that signals not belong to the set categories are incident. Fig.5 shows relation between incident azimuth angle and estimated DOAs in case of 2 waves. In this case, $\theta=0^{\circ}$ for each waves and one wave arrives at delay of 4 symbols compared to the another. It is clear that neurons subject to the nearest categories ignite

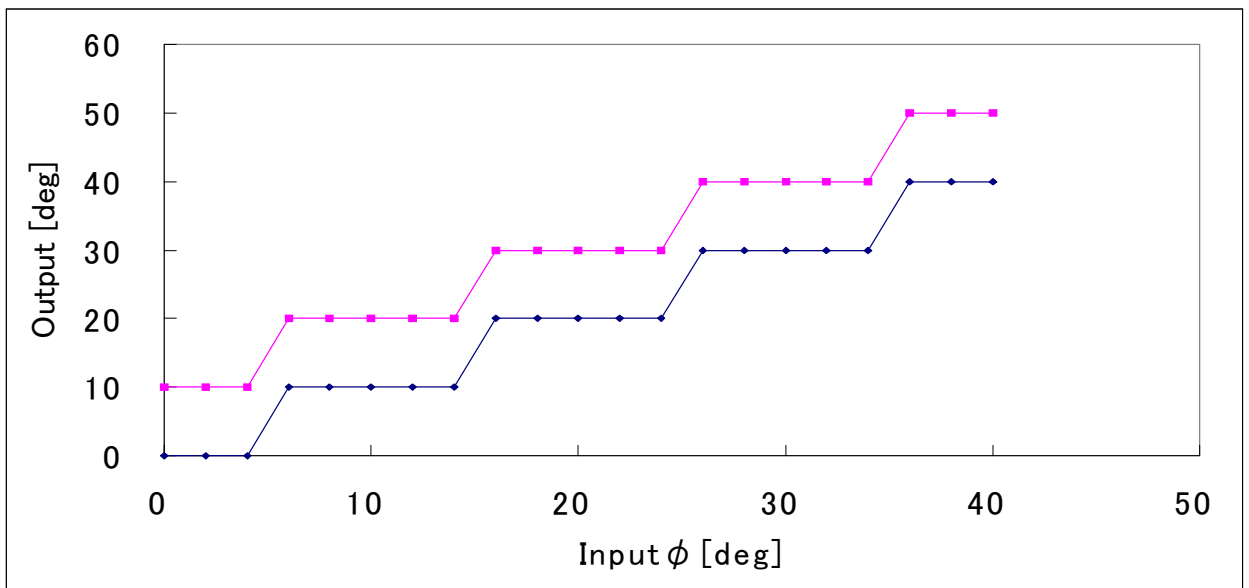

Fig.5 Relation between incident azimuth angle and estimated result ( 2 waves case)

And, we have investigated reliability of the estimated results. We assumed 2 waves with the same amplitude. Parameter set of one wave is established at either of $\left(0^{\circ} 0^{\circ} 0\right.$ symbols $),\left(10^{\circ} 10^{\circ} 0\right.$ symbols $) . .\left(50^{\circ} 50^{\circ} 0\right.$ symbols $)$. Then, the other wave is established 
at all the rest of categories in order. We have trial sets of 179x6 in the end. Number of wrong estimating results has been counted. We also have applied CNR as a parameter to the investigation. Fig.6 shows the results. In this figure, black line shows the case that parameters of both waves were able to estimated correctly, and red line shows the case that parameters of only one wave were able to estimated.

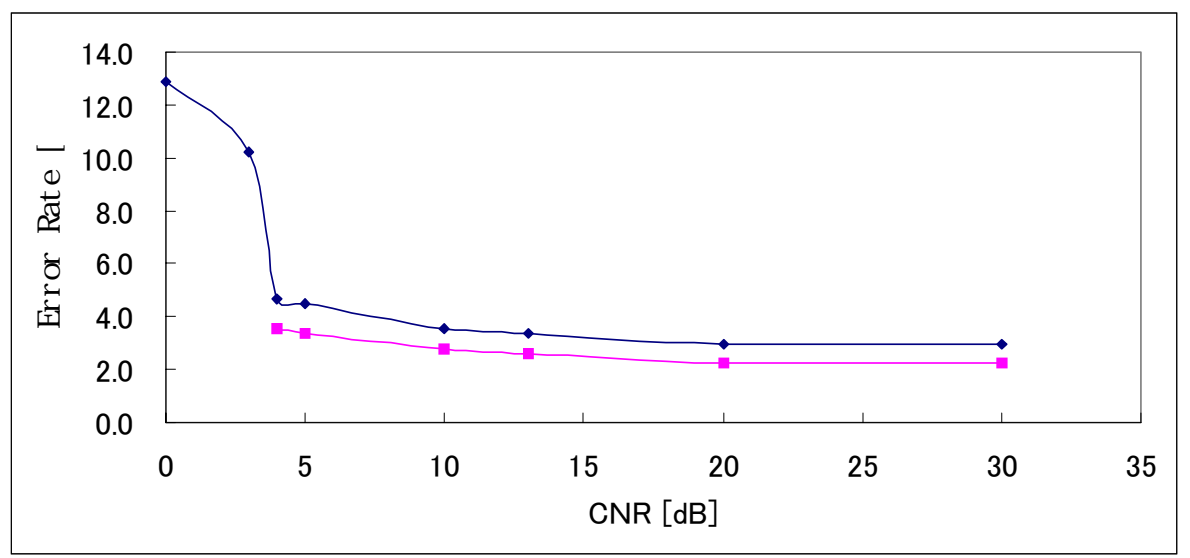

Fig.6 Reliability of estimation

When CNR is better than $10 \mathrm{~dB}$, the error rate is less than $4 \%$. However, it does not fall less than $3.5 \%$ even if CNR was better than $30 \mathrm{~dB}$. In the wrong results, there were some case that one middle neuron ignites. For example, a neuron subject to $\left(10^{\circ} 30^{\circ} 0\right.$ symbols) ignites when 2 waves with $\left(10^{\circ} 40^{\circ} 0\right.$ symbols $)$ and $\left(10^{\circ} 20^{\circ} 0\right.$ symbols $)$ are incident. Though we can improve the reliability by increasing number of parameters $\left(\mathrm{M}_{1}, \mathrm{M}_{2}, \mathrm{~N}\right)$, the calculating load and hardware size increase.

\section{Conclusions}

We have proposed a new algorithm to estimate coupled azimuth, elevation angle and delay of multiple incident waves. It is applied Hopfield neural network, which is not need to learn preliminary. We have plans to expand to coupled azimuth, elevation angle, delay and relative power estimation and to improve reliability of estimation without expansion of hardware size.

\section{References}

[1] A. H. El. Zooghby, C. G. Christodoulou and M. Gergioulous, "Performance of Radial-Basis Function Networks for Direction of Arrival Estimation with Antenna Arrays," IEEE Transactions on Antennas and Priopagation, Vol.45, No.11, Nov.1997, pp.1611-1617.

[2] K. Goto, M. Hirari and M. Hayakawa, "Direction Finding of Distributed Man-Made Noise Source Using Artificial Neural Networks," T. IEE Japan, Vol.118-C, No.3, 1998, pp.442-447.

[3] M. T. Hagan, H. B. Demuth and M. H. Beale, "Neural Network Design," PWS Publishing Company, 1995. 